\title{
A Hybrid Approach for Detection and Classification of the Defects on Printed Circuit Board
}

\author{
Swagata Ray \\ School of Education Technology \\ Jadavpur University \\ Kolkata
}

\author{
Joydeep Mukherjee \\ School of Education Technology \\ Jadavpur University \\ Kolkata
}

\begin{abstract}
Printed Circuit Board (PCB) is the most important component of the electronic industry and thus, the electronic massproduction facilities make an attempt to achieve Printed Circuit Board with $100 \%$ quality assurance. To achieve Printed Circuit Board with $100 \%$ quality assurance i.e. to produce zero-defect Printed Circuit Board, inspection method is the vital process during manufacturing of PCB. This paper presents the Hybrid approach which helps in producing zerodefect PCB by detecting the defects. This approach not only detects the defect but also classifies and locates the defects. The hybrid approach uses referential and non-referential methods to analyze the Printed Circuit Board and this approach proposed an algorithm which involves Image Representation, Image Comparison, and Image Segmentation. The algorithm was tested with mass number of images of Printed Circuit Board. The experimental result shows that higher quality assurance is obtained than the previously implemented algorithms.
\end{abstract}

\section{General Terms}

Defect Detection, Defect Classification, Defect Localization

\section{Keywords}

Printed Circuit Board, Hybrid Approach, Referential and Non-referential methods, Image Comparison

\section{INTRODUCTION}

In the modern world of technology, all the technologies are going towards full digital implementation. The electronic products such as mobile phones, tablet PCs, and so on are becoming necessity of our daily life. Thus, the manufacturing of PCB is becoming more and more important. As, it is important to produce a $\mathrm{PCB}$, it is also important to produce PCB without any defects. To produce zero-defect PCB, the inspection process is the important step which is performed in the manufacturing process. Manual Inspection is the common process to identify the defects of PCB but it provides an inefficient and tiring process and it is also very subjective to errors due to the complex structure of the PCB. Nowadays, the technology of computer vision for the detection of the defects on the goods has been highly developed and used in several industry applications. This technology reduces subjective aspects and provides an efficient and a fast process. The Hybrid approach is one of the applications of the computer vision technology which is presented in this paper.

\subsection{Objectives}

The main objective of this paper is to become familiar with the different types of defects that can occur on PCB, to become familiar with the methodologies for detection of the defects and finally applying the methodologies to detect, to classify and to locate the defects on PCB.

\subsection{Types of Defects of PCB to be Detected}

There are mainly two types of defects that can occur during the manufacturing process of $\mathrm{PCB}$ which are as follows:

a) Functional Defects

b) Cosmetic Defects

Functional Defects can seriously affect the performance of the PCB. While the Cosmetic Defects affect the appearance of the PCB. In this paper we are generally concentrated on the Cosmetic defects of the PCB. There are 14 known types of defects for single layer PCBs which are shown in Figure 1.

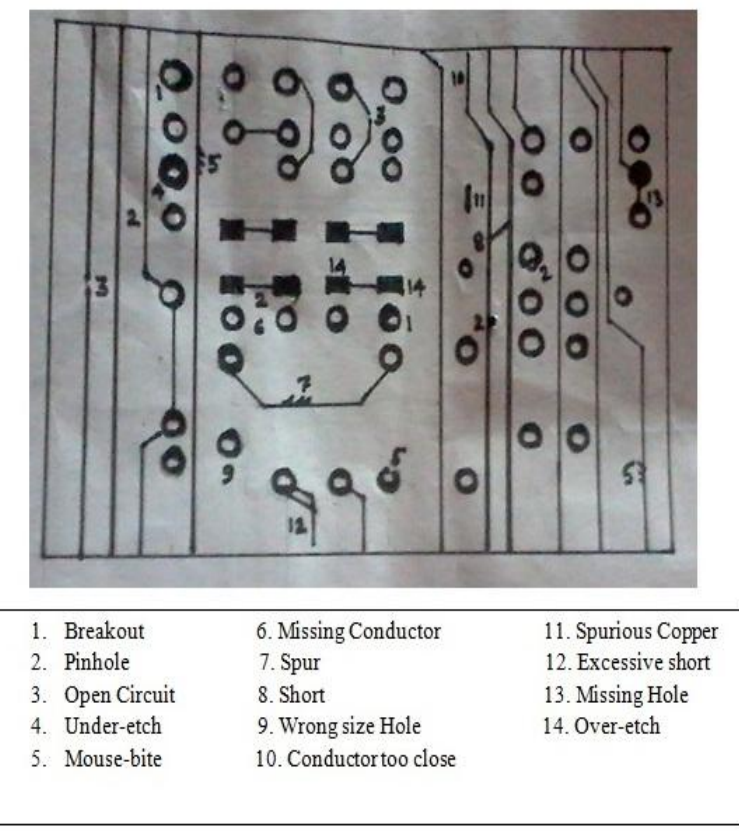

Figure 1: Image of a defected PCB

\subsection{Background Concepts}

This paper introduces the Hybrid approach which involves reference comparison approach and design rule checking approach. The reference comparison approach is based on the comparison between the image of the PCB to be tested and that of an ideal PCB which has pre-defined design specifications. The Design Rule Checking (DRC) method checks if all patterns and spaces of PCB surface meets or violates common knowledge, which is called the design rule. The hybrid approach merges the advantages of the reference 
comparison method and the Design Rule Checking (DRC) method to produce the efficiency of the system.

\section{LITERATURE SURVEY}

[1] proposed an algorithm which includes several image processing operations such as image subtraction, image adding, logical XOR and NOT, and flood fill operator. But, the limitations of this algorithm are as follows:

1. This algorithm is developed to work with binary images only, whereas the output from the cameras is in grayscale format

2. Occurrence of the unwanted noise.

[2] proposed an algorithm which involves comparing a PCB standard with a PCB image, using a simple subtraction algorithm. The main difficulty arises in the pre-processing technique.

[3] proposed a non-contact reference based image processing approach for defect detection and classification. But, in this approach there are many limitations which are as follows:

1. Unwanted images were also generated by noise during gray-scale to binary conversion.

2. Pin-hole defect was ignored.

[4] proposed an approach where a standard PCB image is compared with the inspected PCB image to detect defects by using simple subtraction algorithm. But, this approach has several limitations which are as follows:

1. Some of the Bare PCB defects such as open circuit, short circuit, incorrect dimensions, missing conductor etc. cannot be detected by this Image Subtraction method.

2. Orientation of tested image must be same as the standard image.

[5] proposed an approach where an automatic machine vision system inspects the missing components of PCB by comparing with the standard PCB. The limitations of this approach are as follows:

1. The proposed system is not the generalized system but a dedicated system for a particular PCB and can be optimized to a level.

[6] proposed an approach where the defects on PCB are detected and the effect of noise is observed in this approach.

[7] proposed an approach where image processing algorithm is modified by applying a segmentation process for defect detection on PCB. This approach uses template and test images of single layer, bare, gray-scale computer generated PCBs.

[8] proposed an algorithm which makes the use of image subtraction method for defect detection and kNN classification algorithm is used for the classification of the defects. This approach presents and analyzes the performance of the proposed inspection algorithm.

[9] proposed an algorithm to group all 14 defects found on PCB into 5 Groups. This method has some drawbacks like during computation of defect detection and implementation, this operation brings the unwanted noise due to misalignment along with it.

[10] proposed an approach which separates the defects in larger groups into smaller groups.

\section{REASONS FOR WRITING THIS PAPER}

The work which is presented in this paper is an extension of the existing works to enhance the basic approaches of detecting the defects of PCB with some own implementations. Due to the limitations of the existing work like defect localization, degradation of quality of image due to the occurrences of noise, this approach is proposed which provides:

$\checkmark \quad$ Detection of all possible defects.

$\checkmark$ Classification of the defects into groups.

$\checkmark$ Localization of the defects on PCB

$\checkmark$ Production of the output without any degradation of the image.

\section{METHODOLOGIES}

The different image processing methodologies are used to carry out the proposed approach. The used methodologies are described as follows:

\subsubsection{Image Difference Operation}

Image difference is used to compare the images pixel-by-pixel by using XOR logic operator.

\subsubsection{NOT operator}

NOT operator is used to complement the image.

\subsubsection{Flood-fill operator}

By applying the flood-fill operator the white fill of the hole is changed to black pixel. The produced output contains no hole, because the hole is filled with the black pixel. This output image will be used in image difference or subtraction operations for defect detection.

\subsubsection{Image Subtraction}

Image subtraction method uses the concept of simple subtraction and produces output according to different conditions as shown in Table 1 . In this work, both images of template image and defective image are compared pixel by pixel. The subtract operation produces either negative or positive pixel value.

Table 1: Conditions for image subtraction

\begin{tabular}{|l|l|}
\hline Condition & Output \\
\hline If $1-0=1$ & Positive pixel image \\
\hline If $0-1=-1$ & Negative pixel image \\
\hline
\end{tabular}

\subsubsection{Image Addition}

Image addition is a method for the two images into one image. In this paper, this operator combines the defects from one group with the defects from another group into one image containing all possible defects. This operation is possible by using OR logic operator.

\subsubsection{Thresholding}

In this method, a gray scale image is converted into a binary image by choosing a gray level $\mathbf{T}$ in the original image, and then converting every pixel black or white according to whether its gray value is greater than or less than $\mathbf{T}$.

- A pixel becomes white if its gray level is $>\mathbf{T}$

- A pixel becomes black if its gray level is $<=\mathbf{T}$ 


\subsubsection{Noise Removal}

Noise is considered as the degradation in the image signal, caused by external disturbance. This noise can be removed by Noise Removal procedure. There are different types of noise which can be occurred in the image signal and there are solutions also to remove those noises from the image signal.

\subsubsection{Defect Localization}

The defected patterns are generated in this approach. Now, the defects of the PCB image can be located on PCB. The advantage of defect localization is that it is possible to tell the exact occurrence of the defects. Function regionprops() is used for locating the defects on PCB.

\section{PROBLEM ANALYSIS AND SOLUTION}

\subsection{Proposed Approach}

Printed Circuit Boards are the most common method of assembling modern electronic circuits. During the manufacturing of PCB many defects are introduced which are harmful to the performance of the circuit. Generally, these defects are identified by the manual inspection. But, with the advancement of computer technology digitization in every field, the defects are detected by computer vision technology. A variety of ways has been established to detect the defects found on PCB. But, there are many limitations in the existing work. In this work, the prime importance has given to the quality of the PCB image whether it is synthetically created or the PCB image clicked by the digital camera by removing the unwanted noise from the images. This proposed approach also detects the defects and group them for classifying the defects. The proposed approach involves into five steps which are as follows:

1. The first approach is to read the sample image of the non-defective PCB and the sample image of the PCB to be tested.

2. The second approach is to find out the differences between the images.

3. The third approach is to find out the properties of the differences.

4. The fourth approach is to find the maximum matching entry.

5. The fifth approach is to display the output.

\subsection{Work-Flow of the Proposed Approach}

The work involves in two datasets. Among the two datasets, one dataset is the Template Image i.e. the standard nondefective PCB image which is shown in the Figure 2 with which the other dataset is compared for error detection. The other dataset is the test image i.e. the image of PCB with more or less defects which is shown in the Figure 3 and this image is compared with the template image to find defects on the test image. The work proposes Hybrid Approach. The Hybrid Approach involves in several steps which are explained below in details:

Step 1: At first, the template image and the test image are selected.

Step 2: Then the threshold values of both the images are determined for converting them from gray-scale to binary. Gray-scale images with levels varying between 0 and 255 are converted into binary images with only two levels; 0 s and $1 \mathrm{~s}$. This is to simplify the further process.

Step 3: After the completion of conversion, it is necessary to check whether there is any unwanted noise in the images. If there is any unwanted noise, then the Noise-Removal procedure is performed. It is observed that during the conversion, the degradation of the quality of the images takes place due to the occurrence of unwanted noise in the images and thus this procedure is used to improve the quality of the image.

Step 4: Then the noise-free binary converted images of the template image and the test image are subjected to image subtraction to produce negative image and positive image. Positive image is the result of subtracting the test image from the template image, while, the negative image is the result of subtracting the template image from the test image.

Step 5: Then not operator and Flood-fill operator are applied to the binary converted images of the template image and the test images separately to produce $\mathrm{A} 1$ and $\mathrm{A} 2$ images and from where the various defect detection algorithms are applied to produce the result of the defects.

\subsection{Flow-chart of the Proposed Approach}

The proposed approach is explained by the following flowchart:

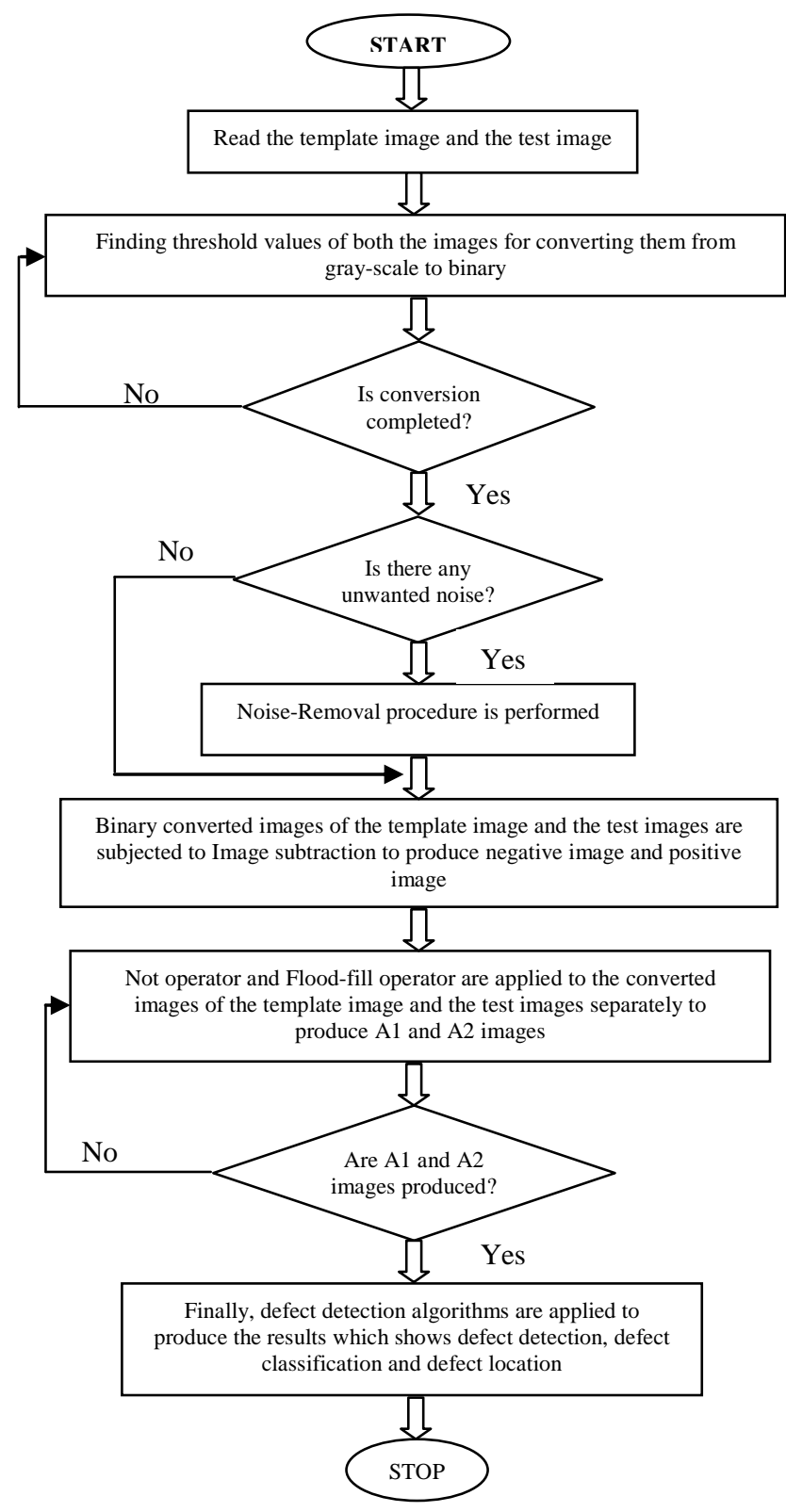




\section{EXPERIMENTAL RESULTS}

\subsection{Datasets used}

The template image and the test image are the two datasets which are used in the proposed approach and they are shown in Figure 2 and Figure 3.

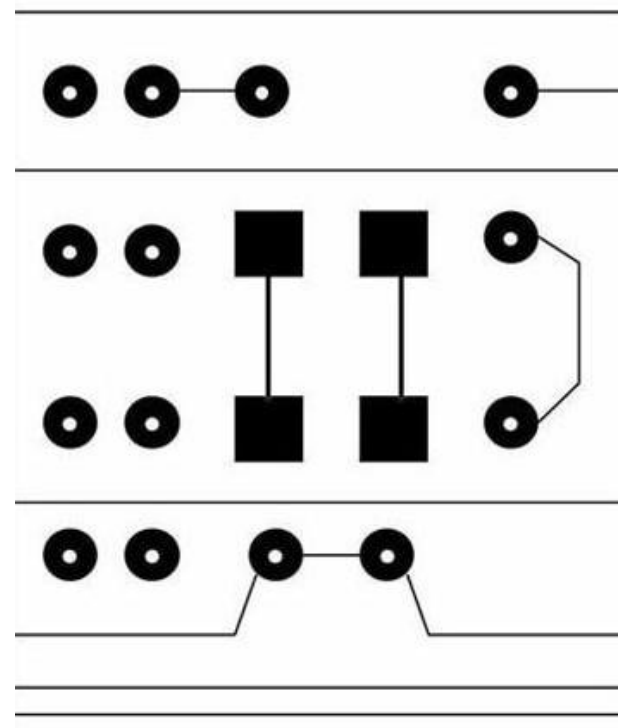

Figure 2: Template Image
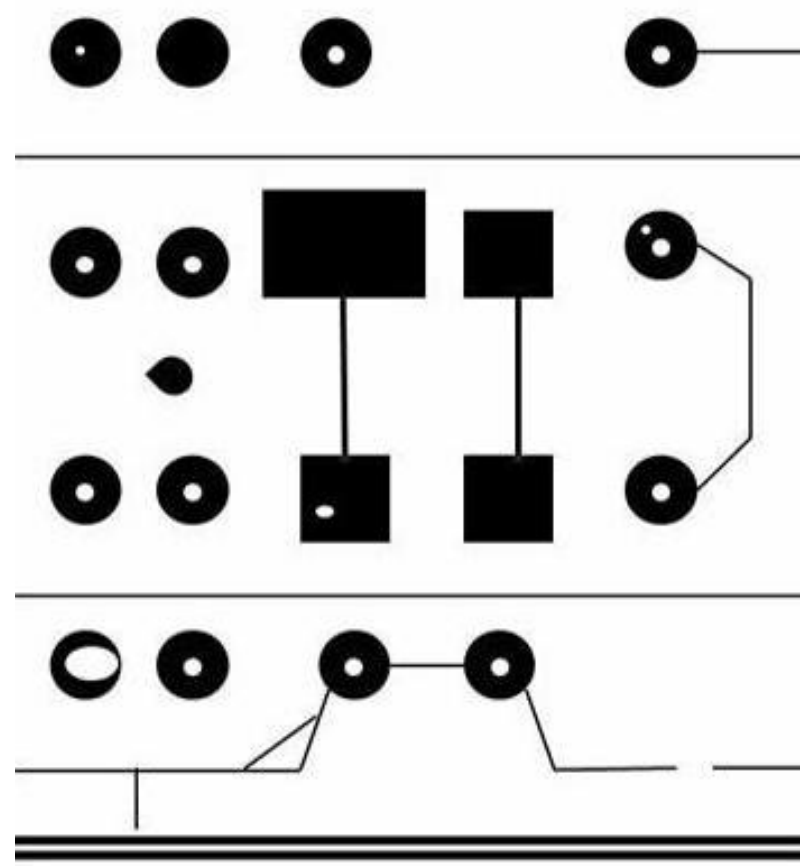

Figure3: Test Image

\subsection{Defect Detection and Classification}

The algorithms are applied to detect and to classify the defects from the test image which are grouped into the followings:

Group 1: missing hole and wrong size hole
Group 2: spur, short, spurious copper, excessive short, underetch, negative, and conductor too close negative.

Group 3: open circuit, mouse bite, over-etch conductor too close positive.

Group 4: under-etch positive.

Group 5: pinhole and breakout

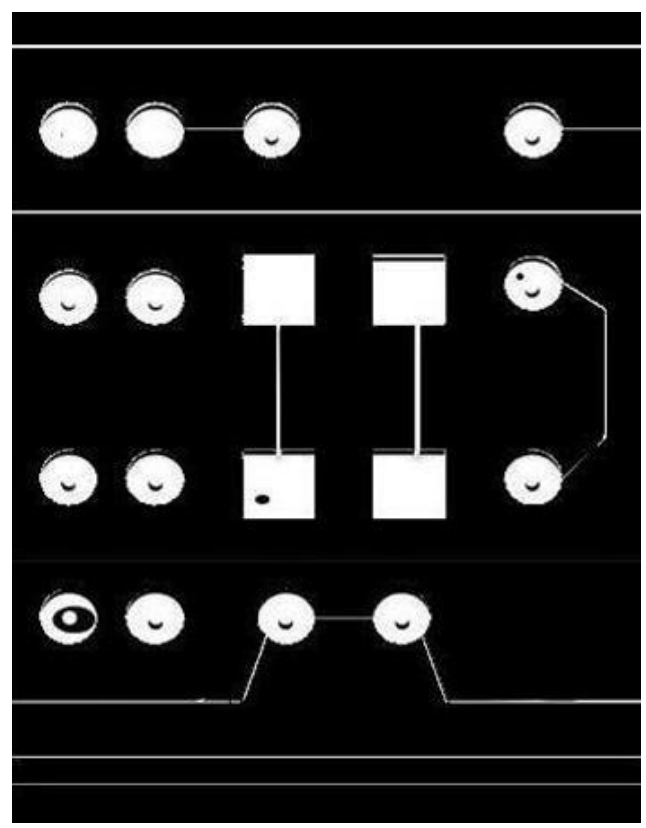

Figure 4: Output showing defects in Group 1
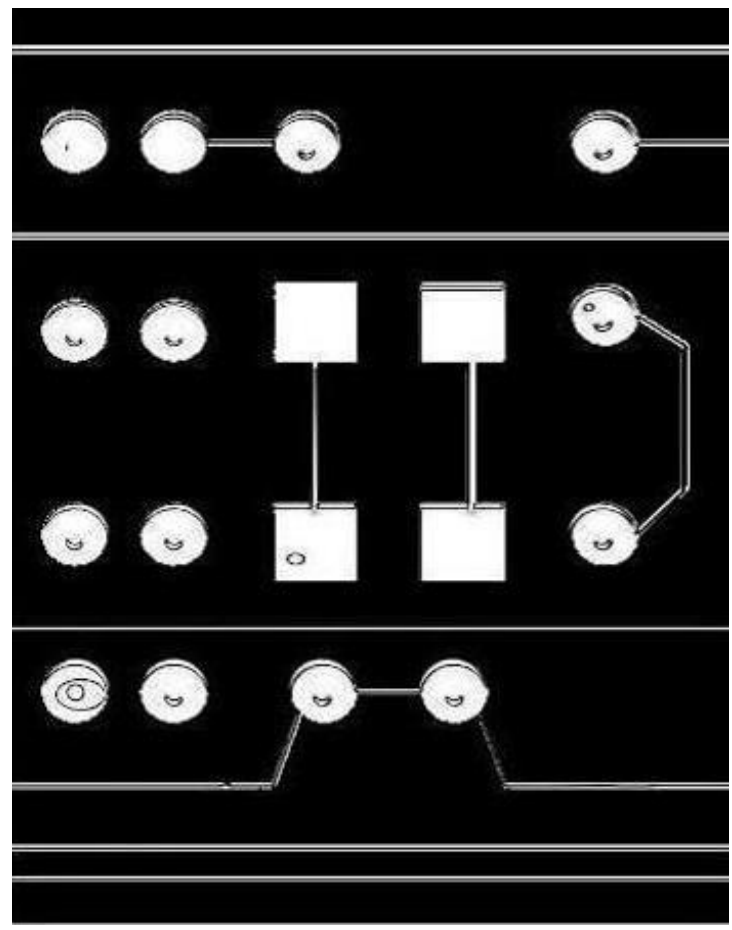

Figure 5: Output showing defects in Group 2 


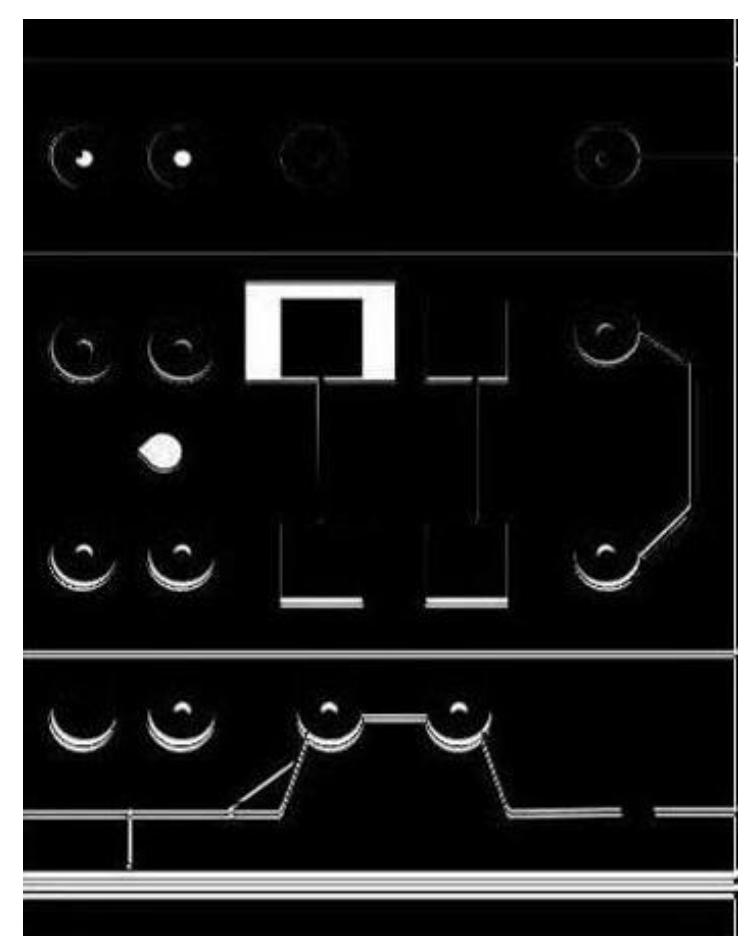

Figure 6: Output showing defects in Group 3

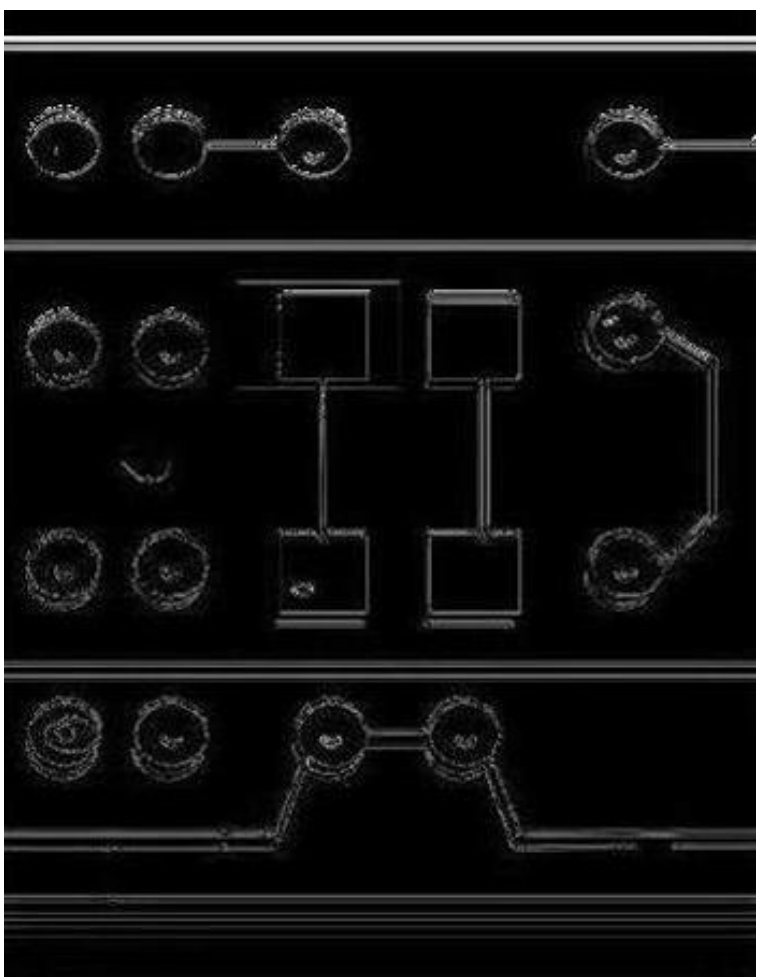

Figure 7: Output showing defects in Group 4

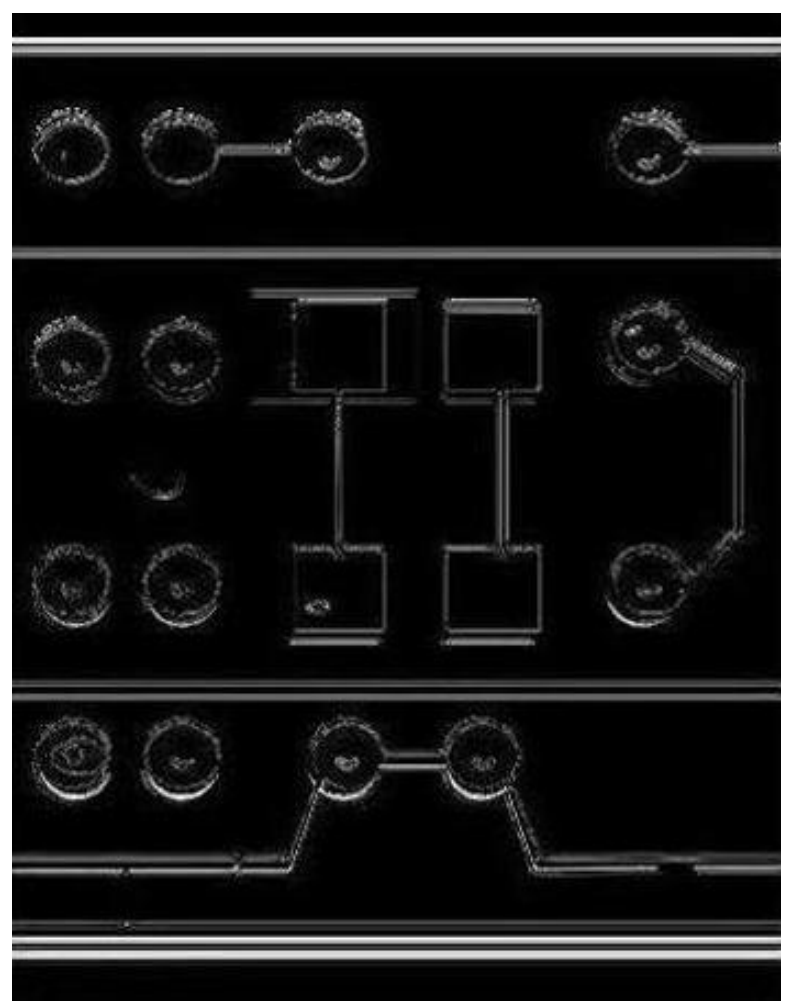

Figure 8: Output showing defects in Group 5

\subsection{Defect Localization on PCB by} Comparing the Template Image and the Test Image

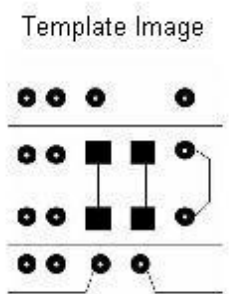

Test Image

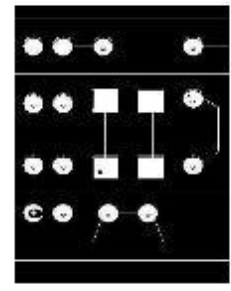

Defects Detected

Location of Defect Detected
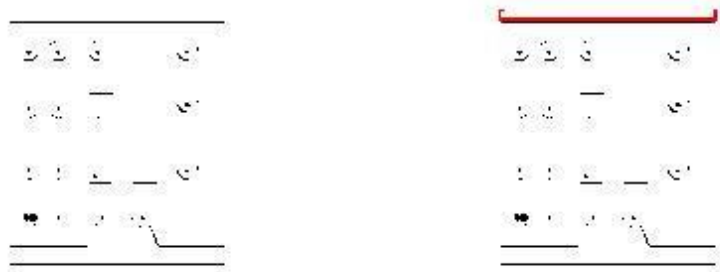

Figure 9: Image showing location of defects in Group 1 


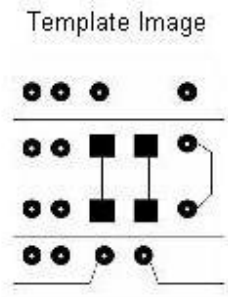

Defects Detected

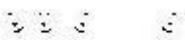

$\therefore--\therefore$

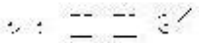

$\therefore \therefore$. .

$+\frac{1}{2}$

Figure 10: Image showing location of defects in Group 2
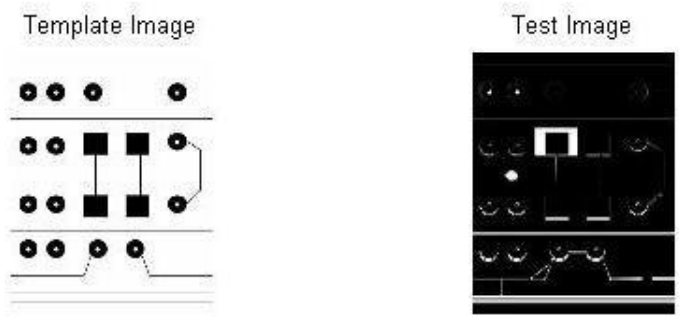

Defects Detected

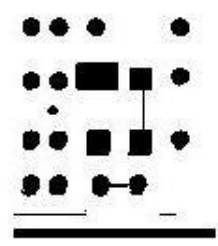

Location of Defect Detected

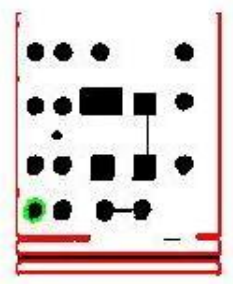

Figure 11: Image showing location of defects in Group 3
Template Image

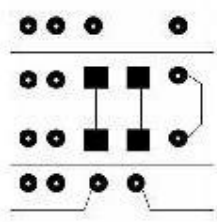

Defects Detected

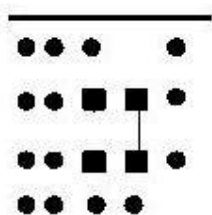

Test Image

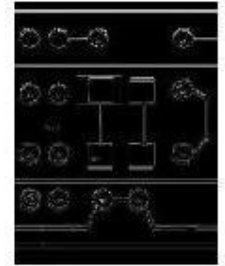

Location of Defect Detected

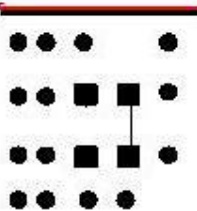

Figure 12: Image showing location of defects in Group 4
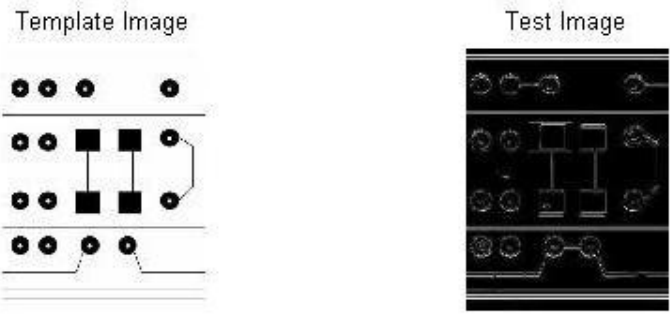

Defects Detected

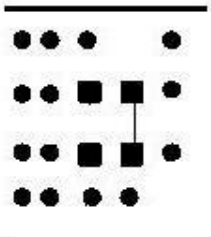

Location of Defect Detected

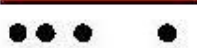

- $\square$ -

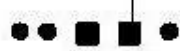

$\rightarrow-$

Figure 13: Image showing location of defects in Group 5

\section{Difference between the Existing Approach and the Proposed Approach}

\begin{tabular}{|l|l|}
\hline \multicolumn{1}{|c|}{ Existing approach } & \multicolumn{1}{|c|}{ Proposed approach } \\
\hline $\begin{array}{l}\text { Used reference comparison } \\
\text { method. }\end{array}$ & $\begin{array}{l}\text { Uses hybrid method which involves } \\
\text { in both reference comparison } \\
\text { method and design rule checking } \\
\text { method. }\end{array}$ \\
\hline $\begin{array}{l}\text { Fails to detect defect at noise } \\
\text { level } 0.01,0.02,0.03 .\end{array}$ & $\begin{array}{l}\text { Successful to detect defect at noise } \\
\text { level } 0.01,0.02,0.03 .\end{array}$ \\
\hline Defects are not localized on PCB. & Defects are localized on PCB. \\
\hline
\end{tabular}

\section{CONCLUSION AND FUTURE SCOPE}

The work is successfully implemented on synthetically created image of PCB to detect, to classify and to locate the defects on PCB. By the proposed algorithm in this research work, the defects are detected and classified into five Groups. By comparing, the research work with the existing work, it is observed that this presented work is giving better result than the existing works, because this work produces the output with no degradation of the quality of the image.

\subsection{Future Scope}

- The approach shown in this paper has implemented on the synthetically created images of PCB only. But, this proposed algorithm can be implemented on the digital photos of PCB which are randomly clicked by digital camera.

- This approach is mainly focused on detecting the defects of PCB after the printing process of the manufacturing process is performed. But, this proposed algorithm can also be used to detect the defects before the printing process of the manufacturing process of PCB. 


\subsection{Limitations}

In this work, the template image and the test image are taken of equal sizes. If the images are not taken of equal sizes then the complexity will arise for applying the proposed algorithm.

\section{ACKNOWLEDGEMENT}

I feel extremely glad in presenting this paper. I convey my gratitude to my project guide Mr.Joydeep Mukherjee for his timely help and encouragement in carrying out this work.

\section{REFERENCES}

[1] Noor Khafifah Khalid, Zuwairie Ibrahim, Mohamad Shukri, Zainal Abidin, "An Algorithm To Group Defects On Printed Circuit Board For Automated Visual Inspection", International Journal of Systems, Science and Technology, Vol.9, No.2, May 2008.

[2] Fabiana R. Leta, Flavio F. Feliciano, Flavius P.R. Martins, "Computer Vision System For Printed Circuit Board Inspection", ABCM Symposium Series in Mechatronics, Vol. 3 - pp.623-632, 2008.

[3] S.H Indera Putera, Z, Ibrahim, "Printed Circuit Board Defect Detection Using Mathematical Morphology and Matlab Image Processing Tools", International Conference on Education Technology and Computer, 2010 .

[4] Ajay Pal Singh Chauhan, Sharat Chandra Bhardwaj, "Detection of Bare PCB Defects by Image Subtraction Method using Machine Vision", Proceedings of the World Congress on Engineering, Vol II, WCE 2011, July $6-8,2011$, London, U.K.
[5] Sanveer Singh, Manu Bharti, "Image Processing Based Automatic Visual Inspection System For PCBs", IOSR Journal of Engineering, ISSN: 2250-3021 Volume 2, Issue 6, June 2012, PP 1451-1455.

[6] Sonal Kaushik, Javed Ashraf, “Automatic PCB Defect Detection Using Image Subtraction Method, International Journal of Computer Science and Network", International Journal of Computer Science and Network, Volume 1, Issue 5, October 2012.

[7] Malge P.S., Nadaf R.S., "PCB Defect Detection, Classification and Localization Using Mathematical Morphology and Image Processing Tools", International Journal of Computer Applications (0975-8887), Volume: 87-No.9, February, 2014.

[8] Prachi P. Londe, S.A. Chavan, "Automatic PCB Defects Detection and Classification using Matlab", International Journal of Current Engineering and Technology, Vol.4, No.3, June, 2014

[9] Kaur Kamalpreet, Kaur Beant, "PCB Defect Detection and Classification Using Image Processing", International Journal of Engineering Research in Management and Technology, ISSN: 2278-9359, Volume-3, Issue-8, August, 2014.

[10] Jagadish.S.Jakati, Sidramayya S Matad, "PCB Defect Detection Based on Pattern Matching and Segmentation Algorithm", International Journal of Advanced Research in Computer and Communication Engineering, Vol.3, Issue 9, September, 2014. 